\title{
EDITORIAL
}

\section{The prevalence of bipolar disorders in the general population: a growing trending topic?}

\author{
Vicent Balanzá-Martínez, ${ }^{1,2,3,4}$ Rafael Tabarés-Seisdedos ${ }^{2,3,5}$ \\ ${ }^{1}$ Teaching Unit of Psychiatry, La Fe University and Polytechnic Hospital, Valencia, Spain. ${ }^{2}$ Department of Medicine, University of Valencia, \\ Valencia, Spain. ${ }^{3}$ Centro de Investigación Biomédica en Red de Salud Mental (CIBERSAM), Madrid, Spain. ${ }^{4}$ International Society for \\ Nutritional Psychiatry Research (ISNPR), International Society for Nutritional Psychiatry Research (ISNPR), Spain. ${ }^{5}$ Fundación para la \\ Investigación del Hospital Clínico de la Comunidad Valenciana (INCLIVA), Valencia, Spain.
}

In the current issue of Revista Brasileira de Psiquiatria, Clemente et al. ${ }^{1}$ report the first meta-analysis of the prevalence of bipolar disorder (BD) from populationbased studies. Strengths of the manuscript include the use of suitable methods of analysis, reporting both annual and lifetime prevalence rates, and providing separate data for bipolar subtypes I and II. The latter is relevant because type II is not simply a milder or minor form of BD.

This publication is of considerable interest, firstly because it confirms that the frequency of BD in the general population is low and closer to the conservative estimates of $1-2 \%$, as suggested by historical data. ${ }^{2,3}$ It is worth pointing out that these might be underestimates because, compared to longitudinal studies, data from population-based cross-sectional studies may underestimate the real prevalence of $\mathrm{BD} .^{4}$

More importantly, the authors found an increase in prevalence estimates over recent decades, in parallel with changing DSM diagnostic criteria. Lifetime prevalence of BD type I has virtually doubled with each edition from DSM-III to DSM-IV. The increase in type II is only marginally significant, although the number of studies is also lower. This is quite surprising, since the only relevant change in the bipolar section of the DSM over the last three decades was the appearance of BD type II in DSMIII-R, whereas criteria for type I have remained essentially the same. In a conservative explanation, the authors attribute the increase in type I to the use of different instruments, whereas growth in type II frequency would be the result of changing DSM criteria.

However, this meta-analysis leaves some key questions "blowing in the wind." For instance, how much of this growth is real or artifactual? In other words, is it the result of increased awareness by clinicians, more accurate nosologies, broadening of diagnostic criteria, or just another vogue? Recently, there has been much controversy about an epidemic of $\mathrm{BD}$, and psychiatry is facing risks for both bipolar "overdiagnosis" and "underdiagnosis." The results of this paper will surely fuel the debate. Interestingly, if one assumes that a genuine increase in bipolar frequency

Correspondence: Vicent Balanzá-Martínez, Departament de Medicina, Facultat de Medicina, Avda Blasco Ibaãez, 15, 46010, Valencia, Spain.

E-mail: Vicente.Balanza@uv.es has taken place, it would be relatively independent of the gradual expansion of the bipolar phenotype, from the narrowly-defined "classic" manic-depressive illness (BD type I) to the broadly-defined "full bipolar spectrum." The latter may present with higher rates, but is more difficult to capture; therefore, its prevalence has been less examined in community samples.

Another fascinating issue is why increased prevalence in non-clinical samples is more obvious in BD type I. One could expect a similar, or even greater, trend for BD type II. Some reflections about hypomania, which lies between the narrow and broad bipolar phenotypes, merit further discussion. Recognition of mania is straightforward, with exceptionally high concordance rates among clinicians. However, an accurate diagnosis of hypomania is usually difficult in clinical practice - even more so in community surveys! On the one hand, patients certainly do not label hypomania as abnormal. On the other hand, the successive DSM editions have refined the diagnostic criteria for hypomania, but its definition remains controversial. Hypomania has not gone through Robins and Guze's gold-standard criteria to establish the validity and reliability of major mental disorders. ${ }^{3}$ Indeed, its dissection from mania does not rely on quantity or quality of symptoms, which are identical, with the exception of psychotic features. Rather, it is based on the somewhat arbitrary, non-symptom criteria of minimum duration of 7 days, need for hospitalization, and marked functional impairment in the case of mania. ${ }^{5}$ Moreover, marked impairment is not clearly operationalized in DSM. ${ }^{6}$ All of this likely leads to underestimation of the true prevalence of BD type II, especially in the general population. As mentioned by the authors, its prevalence is greater in clinical samples. That also seems to be the case among adolescents, an age group in which lifetime prevalence rates of hypomania can reach $3-4 \%$ in prospective community samples. ${ }^{7}$ This question must await further inquiry, because the present systematic review focused only on adult samples. Future meta-analyses should also reveal whether the inclusion of studies using the ICD diagnostic system would modify the present findings.

Certainly, establishing the prevalence of bipolar and other psychiatric disorders is a complex endeavor. Differences in settings (general population, primary care vs. psychiatric clinics), types of prevalence estimates 
(e.g., point, 12-month, lifetime), assessment instruments, and changing diagnostic criteria may affect prevalence rates and comparisons of studies. These factors may likely account for much of the discrepancy in the literature regarding $\mathrm{BD}$ prevalence. However, defining the boundaries of the phenotype is unique to $\mathrm{BD}$ and represents a major source of this variability, as mentioned above.

$\mathrm{BD}$ is among the leading causes of disability worldwide. Robust and reliable prevalence estimates, such as those from the present meta-analysis, represent a relevant addition to the epidemiology of this disorder and have clinical, socioeconomic, and policy-making implications. For instance, they may help inform planning of health care and services. People with BD deserve more effective treatments, and advancing a better definition, ideally based on a better understanding of BD neurobiology, is a key step. ${ }^{6}$ Clearly, there is substantial room for improvement of our current diagnostic systems. In this regard, recent proposals, such as clinical staging systems ${ }^{8}$ and Research Domain Criteria, ${ }^{9}$ will hopefully move the field forward and improve clinical outcomes.

\section{Acknowledgements}

The authors would like to acknowledge the support of the Spanish Ministry of Economy and Competitiveness, Instituto de Salud Carlos III (ISCIII), and Centro de Investigación Biomédica en Red de Salud Mental (CIBERSAM). This work was sponsored by a grant from Fundación para el Fomento de la Investigación Sanitaria y Biomédica de la Comunitat Valenciana (FISABIO; UGP-14-184) and a national grant that belongs to the Spanish Plan Nacional de I + D + I 2013-2016 (PI14/00894) and is co-funded by the
ISCIII-Subdirección General de Evaluación y el Fondo Europeo de Desarrollo Regional (FEDER).

\section{Disclosure}

The authors report no conflicts of interest.

\section{References}

1 Clemente AS, Diniz BS, Nicolato R, Kapczinski FP, Soares JC, Firmo $\mathrm{JO}$, et al. Bipolar disorder prevalence: a systematic review and metaanalysis of the literature. Rev Bras Psiquiatr. 2015;37:155-61.

2 Goodwin FK, Jamison KR. Manic-depressive illness: bipolar disorders and recurrent depression. 2nd edition. New York: Oxford University Press; 2007.

3 Yutzy SH, Woofter CR, Abbott CC, Melhem IM, Parish BS. The increasing frequency of mania and bipolar disorder: causes and potential negative impacts. J Nerv Ment Dis. 2012;200:380-7.

4 Dell'Aglio JC Jr, Basso LA, Argimon IIL, Arteche A. Systematic review of the prevalence of bipolar disorder and bipolar spectrum disorders in population-based studies. Trends Psychiatry Psychother. 2013;35: 99-105.

5 Parker GB, Graham RK. An evaluation of the DSM-5 rules defining mania and hypomania with identical symptom criteria. J Affect Disord. 2015;170:91-4.

6 Malhi GS, Berk M. Diagnosing bipolar disorder: defining thresholds and setting boundaries. Aust N Z J Psychiatry. 2014;48:500-4.

7 Merikangas KR, Lamers F. The 'true' prevalence of bipolar II disorder. Curr Opin Psychiatry. 2012;25:19-23.

8 Kapczinski F, Magalhães PV, Balanzá-Martinez V, Dias VV, Frangou S, Gama CS, et al. Staging systems in bipolar disorder: an International Society for Bipolar Disorders Task Force Report. Acta Psychiatr Scand. 2014;130:354-63.

9 Insel T, Cuthbert B, Garvey M, Heinssen R, Pine DS, Quinn K, et al. Research domain criteria (RDoC): toward a new classification framework for research on mental disorders. Am J Psychiatry. 2010; 167:748-51. 